\title{
Associations between Stress and Physical Activity in Korean Adolescents with Atopic Dermatitis Based on the 2018-2019 Korea Youth Risk Behavior Web-Based Survey
}

\author{
Sunga Kong ${ }^{1,2} \mathbb{D}$, Jaisun Koo ${ }^{3, *}$ and Seung Kil Lim ${ }^{4, *}$ \\ 1 Department of Clinical Research Design and Evaluation, SAIHST, Sungkyunkwan University, \\ Seoul 06351, Korea; sunga00kong@gmail.com \\ 2 Patient-Centered Outcomes Research Institute, Samsung Medical Center, Seoul 06351, Korea \\ 3 Da Vinci College of General Education, Chung-Ang University, Seoul 06974, Korea \\ 4 Department of Exercise Prescription, Dongshin University, Naju, Jeonnam 58245, Korea \\ * Correspondence: susanna9@hanmail.net (J.K.); es007-0@hanmail.net (S.K.L.); Tel.: +82-2-820-5956 (J.K.); \\ +82-61-330-3384 (S.K.L.); Fax: +82-61-330-3386 (S.K.L.)
}

Received: 18 October 2020; Accepted: 29 October 2020; Published: 5 November 2020

check for updates

\begin{abstract}
This study aimed to confirm the associations between stress and physical activity (PA) in Korean adolescents with atopic dermatitis (AD) based on data from the 2018-2019 Korea Youth Risk Behavior Web-Based Survey. The AD groups were divided into adolescents who were not diagnosed with $\mathrm{AD}$, adolescents who were diagnosed with $\mathrm{AD}$ more than one year ago, and adolescents who were diagnosed with AD within one year. We defined the regular PA group and the non-PA group using the American College of Sports Medicine guidelines for children and adolescents: moderate to vigorous $\mathrm{PA} \geq 5$ times per week, including vigorous $\mathrm{PA} \geq 3$ days per week and muscle strengthening exercises $\geq 3$ times per week. We performed logistic regression analysis to calculate the stress odds ratio (OR) and 95\% confidence interval (CI) by group using model 1, adjusted for age, sex, and body mass index, and model 2, additionally adjusted for drinking, smoking, economic statuses, academic achievement, asthma, and rhinitis. In the group diagnosed with AD within one year, stress was $41 \%$ and $32 \%$ higher according to models 1 (1.41 (1.31-1.52)) and 2 (1.34 (1.20-1.50)), respectively. However, the stress $\mathrm{OR}$ was $30 \%$ lower in adolescents who completed regular PA than in the non-PA group (model 1: 0.71 (0.58-0.87); model 2: 0.68 (0.57-0.84)), even if diagnosed with AD within one year. In conclusion, the stress of adolescents with AD was significantly higher than that of adolescents without AD. The stress was significantly lower in the group with regular PA, and it was more robust in adolescents diagnosed with AD within one year.
\end{abstract}

Keywords: atopic dermatitis; stress; physical activity; exercise; youth

\section{Introduction}

Atopic dermatitis (AD) is a disease characterized by repeated cycles of exacerbation and improvement of itchy eczema [1]. Among adolescents, AD is one of the most common chronic diseases, along with asthma and rhinitis [2]. The incidence of AD in children has increased twoto three-fold over the past 30 years [3,4], with an incidence of more than $20 \%$ among children and adolescents worldwide [5]. In South Korea, the incidence of AD among children and adolescents is currently 13.5\% and increasing [6]. The main symptoms of AD include itching and hypersensitivity to external stimuli and allergens, and these symptoms are known to worsen in adolescence [7]. 
Although the etiology of AD is still unclear, studies have reported that it is caused by an interaction between genetic, environmental, and immunological factors and a dysfunction in the skin barrier [8].

$\mathrm{AD}$ degrades quality of life by affecting an individual's sleep, emotional and mental health, physical activity (PA), and social functioning [9]. In particular, adolescents are known to demonstrate problems in establishing a healthy body image; they have low perceived happiness and high stress, as they undergo esthetic and functional skin changes caused by chronic and repeated inflammatory reactions [10]. Furthermore, adolescents experience negative social and psychological changes, such as depression and anxiety [11,12]. A prior study reported higher suicidal ideation (44\%) and suicide attempts (36\%) among patients with AD compared to their non-AD counterparts [13]. A study of data from the Korea Youth Risk Behavior Web-Based Survey (KYRBWS) VI reported that $46 \%$ of adolescents with a high level of stress and $21 \%$ of adolescents with a moderate level of stress suffer from AD [14]. In light of these studies, AD may not only induce stress, but it may also be caused by stress [11]. Therefore, the stress level in youths with AD is an important factor in terms of the improvement of disease symptoms and treatment, as well as patient mental health.

Among various measures to manage mental health, regular PA is known to contribute to improving physical function and mental stress $[15,16]$. An animal study reported that moderate-intensity aerobic exercise improves the symptoms of AD through immunoregulation [17]. However, no human studies have been reported to date. Moreover, PA in patients with AD has both positive and negative aspects, as it can help improve AD by reducing stress [15,16], but can also exacerbate symptoms of $\mathrm{AD}$ by increasing body temperature and inducing perspiration [18]. Although studies in Korea and abroad have investigated the percentage of adolescents with AD who participate in PA [19,20], none have examined the relationship between stress and PA in adolescents with $\mathrm{AD}$; hence, the impact of PA on stress in adolescents with AD remains unclear. Thus, it is necessary to investigate the relationship between stress and PA in adolescents with AD. This study aimed to investigate the risk of stress in adolescents with $\mathrm{AD}$ and the association between stress and regular PA by using data from the KYRBWS.

\section{Methods}

\subsection{Study Participants}

Raw data from the 2018 (14th) and 2019 (15th) KYRBWS, a government-approved statistical survey examining the health behaviors of middle and high school students in Korea, were analyzed in this study. The raw data were gathered from the Korea Centers for Disease Control and Prevention website after submitting an application outlining the purpose and plan of use and obtaining approval. The KYRBWS was conducted on 7th to 12th graders in 800 middle and high schools in Korea, selected by stratifying the population, allocating the sample, and extracting the sample. A total of 60,040 out of 62,823 (95.6\%) participants completed the KYRBWS-14, and 57,303 out of 60,100 (95.3\%) participants completed the KYRBWS-15. Data from these participants $(N=117,343)$ were used in the final analysis.

\subsection{Measurements}

Participants who answered "No" to the question, "Have you ever been diagnosed with atopic dermatitis (eczema or congenital fever) by a physician?" were included in the non-AD group. Those who answered "Yes" to the same question were additionally asked, "Have you been diagnosed with atopic dermatitis (eczema or congenital fever) by a physician in the past 12 months?" to further divide AD participants into the $\mathrm{AD} \geq 1$ year and $\mathrm{AD}<1$ year groups.

For stress, those who chose "extremely high," "high," or "moderate" to the question, "How is your day-to-day stress level?" were considered to have stress, whereas those who chose "low" or "none" were considered to have no stress.

To define regular PA, participants were asked, "How many days in the past 7 days have you engaged in PA (all types) that increases your heart rate above the normal rate or makes you short 
of breath for at least 60 min a day?" and were divided into the " $\geq 5$ days" or " $<5$ days" categories. Participants were also asked, "How many days in the past 7 days have you engaged in vigorous PA that makes you extremely short of breath or sweat for at least $20 \mathrm{~min}$ a day?" and were divided into the " $\geq 3$ days" or " $<3$ days" categories. Participants were also asked, "How many days in the past 7 days have you engaged in muscle strengthening exercises, such as push-ups, sit-ups, lifting weights, using dumbbells, pull up bar, and parallel bar?" and were divided into the " $\geq 3$ days" or " $<3$ days" categories [21].

For physical characteristics, participants' age, sex, and body mass index (BMI; $\mathrm{kg} / \mathrm{m}^{2}$ ) were examined. Smoking status was determined using the yes-or-no question, "Have you ever smoked at least one cigarette?" and drinking status was determined using the yes-or-no question, "Have you ever had at least one shot of alcohol?" Economic status was determined using the question, "What is your household's economic status?" The responses were grouped into "high" and "middle-high," or into "middle," "middle-low," and "low." Academic achievement was determined using the question, "How were your school grades in the past 12 months?" The responses were grouped into "high" and "middle-high," or into "middle," "middle-low," and "low."

\subsection{Statistical Analysis}

All statistical analyses were performed using STATA version 15.0 (STATA Corp., College Station, TX, USA). A complex sample design was used to compute representative values for the Korean adolescent population. The physical characteristics according to the $\mathrm{AD}$ group (non- $\mathrm{AD}, \mathrm{AD} \geq 1$ year, and $\mathrm{AD}<1$ year) were compared using the mean, standard error, and percentage within each group (\%). The participants' characteristics according to AD groups were examined by the $F$-test of the average between $\mathrm{AD}$ groups (all $\mathrm{AD}$ diagnoses) compared to the non-AD group using a complex samples regression. Differences in smoking status, drinking status, economic status, academic achievement, moderate and vigorous physical activity (MVPA), and muscle strengthening exercises among the AD groups were analyzed with chi-square tests. The stress odds ratio (OR) according to the AD group was compared using multiple logistic regression. The ORs and 95\% confidence intervals (CI) for the AD groups were presented in models 1 and 2 based on adjustment. Model 1 was adjusted for age, sex, and obesity (BMI $<18.5,18.5-22.9$, or $\geq 23$ ), and model 2 was additionally adjusted for drinking status, smoking status, economic status, academic achievement, asthma, and rhinitis. Furthermore, the stress OR according to AD and PA was analyzed using the same method. Statistical significance was set at $\alpha=0.05$ for all analyses.

\section{Results}

\subsection{Participant's Characteristics According to AD Diagnosis}

The participant characteristics of the non- $\mathrm{AD}, \mathrm{AD} \geq 1$ year, and $\mathrm{AD}<1$ year groups are described in Table 1. A total of 8099 out of 117,343 (6.8\%) participants had been diagnosed with AD within one year. The AD group was older and had a higher BMI than the non-AD group $(p<0.001)$. The AD $<1$ year group had a higher percentage of respondents who reported smoking and drinking but lower MVPA and muscle strengthening exercises compared to the non-AD group (Table 1). 
Table 1. Participant characteristics by atopic dermatitis diagnosis.

\begin{tabular}{|c|c|c|c|c|}
\hline & $\begin{array}{c}\text { Non-AD } \\
(n=89,863)\end{array}$ & $\begin{array}{l}\mathrm{AD} \geq 1 \text { year } \\
(n=19,381)\end{array}$ & $\begin{array}{c}\mathrm{AD}<1 \text { year } \\
(n=8099)\end{array}$ & $p$-Value \\
\hline Age, years & $15.1 \pm 0.0$ & $15.3 \pm 0.0^{\mathrm{a}}$ & $15.2 \pm 0.3^{\mathrm{a}, \mathrm{b}}$ & $<0.001$ \\
\hline Male sex (\%) & 54.3 & 44.8 & 43.8 & $<0.001$ \\
\hline Body mass index, $\mathrm{kg} / \mathrm{m}^{2}$ & $21.3 \pm 0.0$ & $21.4 \pm 0.0$ & $21.6 \pm 0.1^{\mathrm{a}, \mathrm{b}}$ & $<0.001$ \\
\hline Grade $(\%)$ & & & & $<0.001$ \\
\hline Middle school & 48.1 & 43.5 & 45.3 & \\
\hline High school & 52.9 & 56.5 & 54.7 & \\
\hline Smoking status, yes (\%) & 13.9 & 12.5 & 16.5 & $<0.001$ \\
\hline Alcohol status, yes (\%) & 40.3 & 42.3 & 43.3 & $<0.001$ \\
\hline Household income (\%) & & & & $<0.001$ \\
\hline High or medium-high & 40.7 & 38.9 & 39.0 & \\
\hline Medium, medium-low, or low & 59.3 & 61.1 & 61.0 & \\
\hline Academic achievement (\%) & & & & $<0.001$ \\
\hline High or medium-high & 38.0 & 40.6 & 37.7 & \\
\hline Medium, medium-low, or low & 62.0 & 59.4 & 62.3 & \\
\hline Moderate PA (\%) & & & & $<0.001$ \\
\hline$<5$ days per week & 85.2 & 87.7 & 86.0 & \\
\hline$\geq 5$ days per week & 14.8 & 12.3 & 14.0 & \\
\hline Vigorous PA (\%) & & & & $<0.001$ \\
\hline$<3$ days per week & 64.2 & 68.4 & 66.3 & \\
\hline$\geq 3$ days per week & 35.8 & 31.5 & 33.7 & \\
\hline Muscle strengthening exercises (\%) & & & & $<0.001$ \\
\hline No or 1 time per week & 76.5 & 80.8 & 78.5 & \\
\hline$\geq 3$ days per week & 23.5 & 19.2 & 21.5 & \\
\hline Regular PA (\%) & 8.7 & 6.9 & 7.9 & $<0.001$ \\
\hline
\end{tabular}

Data are presented as mean \pm standard error, percent (\%). Non-AD: The group of adolescents not diagnosed with atopic dermatitis (AD). $\mathrm{AD} \geq 1$ year: The group of adolescents diagnosed with $\mathrm{AD}$ more than one year ago. $\mathrm{AD}<1$ year: The group of adolescents diagnosed with $\mathrm{AD}$ within one year. PA: Physical activity. Regular physical activity (\%): Moderate and vigorous physical activity (MVPA) $\geq 5$ times per week, including vigorous PA $\geq 3$ days per week and muscle strengthening exercises $\geq 3$ times per week. ${ }^{a} p<0.05$ compared to the non-atopic dermatitis group. ${ }^{\mathrm{b}} p<0.05$ compared to the AD group.

\subsection{Stress $O R$ According to $A D$ and $P A$}

Compared to the non-AD group, the AD $<1$ year group had a significantly higher OR for stress, at $1.41(1.31-1.52)$ in model 1 and $1.34(1.20-1.50)$ in model $2(p<0.001)$. However, the $A D \geq 1$ year group only had a significantly higher OR in model 1 , at $1.17(1.11-1.23)$, but not in model 2, at $1.06(0.98-1.14)$ (Table 2).

Table 2. Odds ratios of stress status by atopic dermatitis diagnosis.

\begin{tabular}{lcccc}
\hline & $\begin{array}{c}\text { Non-AD } \\
(\boldsymbol{n}=\mathbf{8 9 , 8 6 3 )}\end{array}$ & $\begin{array}{c}\text { AD } \geq \mathbf{1} \text { year } \\
(\boldsymbol{n}=\mathbf{1 9 , 3 8 1})\end{array}$ & $\begin{array}{c}\text { AD < 1 year } \\
(\boldsymbol{n}=\mathbf{8 0 9 9 )}\end{array}$ & $p$-Value \\
\hline${ }^{\mathrm{a}}$ Model 1 OR (95\% CI) & Reference & $1.17(1.11-1.23) *$ & $1.41(1.31-1.52) *$ & $<0.001$ \\
${ }^{\mathrm{b}}$ Model 2 OR (95\% CI) & Reference & $1.06(0.98-1.14)$ & $1.34(1.20-1.50) *$ & $<0.001$ \\
\hline
\end{tabular}

OR: Odds ratio; CI: Confidence interval. a Model 1: Adjusted for age, sex, and body mass index $(<18.5$, $18.5-22.9$, or $\left.\geq 23 \mathrm{~kg} / \mathrm{m}^{2}\right)$. ${ }^{\mathrm{b}}$ Model 2: Further adjusted for household income (<medium-high or $\geq$ medium-high), academic achievement ( $<$ medium-high or $\geq$ medium-high), smoking status, alcohol status, and other diseases (asthma or allergic rhinitis). Non-AD: The group of adolescents not diagnosed with atopic dermatitis (AD). $\mathrm{AD} \geq 1$ ear: The group of adolescents diagnosed with $\mathrm{AD}$ more than one year ago. $\mathrm{AD}<1$ year: The group of adolescents diagnosed with AD within one year. * Significantly different from the reference group $(p<0.05)$.

\subsection{Stress OR According to AD and PA}

The stress OR among the AD groups was compared according to PA. Those who engaged in regular PA had a significantly lower stress OR than in the non-AD group, with $0.79(0.75-0.84)$ in model 1 and $0.76(0.72-0.81)$ in model $2(p<0.001)$. The OR for stress was also significantly lower in the 
$\mathrm{AD} \geq 1$ year group, with $0.83(0.72-0.97)$ in model 1 and $0.80(0.70-0.93)$ in model $2(p<0.05)$. Finally, the OR for stress was also significantly lower in the AD $<1$ year group, with $0.71(0.58-0.87)$ in model 1 and $0.68(0.57-0.84)$ in model $2(p<0.001)$ (Table 3).

Table 3. Odds ratios of stress status by atopic dermatitis diagnosis and physical activity.

\begin{tabular}{cccc}
\hline & $\begin{array}{c}\text { Non-AD } \\
(n=89,863)\end{array}$ & $\begin{array}{c}\text { AD } \geq 1 \text { year } \\
(n=19,381)\end{array}$ & $\begin{array}{c}\text { AD }<\text { 1 year } \\
(n=8099)\end{array}$ \\
\hline${ }^{\text {a }}$ Model 1 OR (95\% CI) & Reference & Reference & Reference \\
Non-PA & $0.79(0.75-0.84) *$ & $0.83(0.72-0.97) *$ & $0.71(0.58-0.87) *$ \\
Regular PA & $<0.001$ & 0.015 & 0.001 \\
$p$-Value & & & Reference \\
Non-PA & Reference & Reference & $0.68(0.57-0.84) *$ \\
Regular PA & $0.76(0.72-0.81) *$ & $0.80(0.70-0.93) *$ & $<0.001$ \\
p-Value & $<0.001$ & 0.003 & \\
\hline
\end{tabular}

OR: Odds ratio; CI: Confidence interval; PA: Physical activity. ${ }^{a}$ Model 1: Adjusted for age, sex, and body mass index $\left(<18.5,18.5-22.9\right.$, or $\left.\geq 23 \mathrm{~kg} / \mathrm{m}^{2}\right)$. ${ }^{\mathrm{b}}$ Model 2: Further adjusted for household income $(<$ medium-high or $\geq$ medium-high), academic achievement ( $<$ medium-high or $\geq$ medium-high), smoking status, alcohol status, and other diseases (atopic dermatitis or allergic rhinitis). Non-AD: The group of adolescents not diagnosed with atopic dermatitis (AD). $A D \geq 1$ year: The group of adolescents diagnosed with $A D$ more than one year ago. $\mathrm{AD}<1$ year: The group of adolescents diagnosed with $\mathrm{AD}$ within one year. Regular physical activity (\%): MVPA $\geq 5$ times per week, including vigorous PA $\geq 3$ days per week and muscle strengthening exercises $\geq 3$ times per week. * Significantly different from the reference group $(p<0.05)$.

\section{Discussion}

This study analyzed data from the KYRBWS obtained from the Korean adolescent population. The risks of stress in adolescents diagnosed with $\mathrm{AD}$ within one year were $41 \%$ and $34 \%$ higher than in non-AD adolescents in the two adjusted models, respectively. However, among those diagnosed with $\mathrm{AD}$, adolescents who engaged in regular PA had a significantly lower stress risk compared to the non-PA adolescent group, and these results were more robust among adolescents diagnosed with AD within one year.

In this study, we showed that stress was significantly higher in the AD group than in the non-AD group. These results are consistent with those of prior cross-sectional [14,22] and longitudinal studies [23], where AD and stress were reported to be associated. Park and Kim (2016) observed that the risk for severe stress was twice as high in adolescents with AD compared to their healthy counterparts [22], and Kwon (2013) reported that male adolescents with severe stress had a 46\% higher risk of $\mathrm{AD}$ [14]. Our results also demonstrated that $\mathrm{AD}$ and stress are associated, supporting previous findings. In general, AD symptoms and mental stress affect one another in a vicious cycle. Some studies have reported that stress affects the development and progression of AD and exacerbates its symptoms by stimulating the hypothalamic-pituitary-adrenal axis [24]. According to recent research, the evaluation of psychoneuroimmunologic factors and emotional stress is an important component of treatment, in addition to interventions, to improve mental health and reduce stress for patients with $\mathrm{AD}$, as well as to improve patients' health and to markedly improve skin symptoms [24]. Thus, health behavior-related factors that may contribute to improving stress in adolescents with AD should be considered.

In this study, regular PA was associated with a lower risk of stress compared to the non-PA group. The results from previous studies confirmed that regular PA can help improve physical and psychological health among children and adolescents [25,26]. In a recently reported model- and data-based research results, it was reported that PA is related to adolescent mental health among young British adolescents [27]. In that study, regular PA was linked with better mental health, characterized by higher self-esteem and fewer internalizing problems $(\beta=-0.24 ; 95 \% \mathrm{CI},-0.27$ to -0.20$)$. As shown in the systematic review by Mücke et al., high PA reduces stress-related cortisol and heart rate responsiveness, even when participants were exposed to high stress levels [28]. They reviewed smaller increases in 
anxiety and smaller decreases in calmness in participants with regular PA. In our study, even the group diagnosed with $\mathrm{AD}$ within one year also demonstrated a 30\% lower risk of stress when they engaged in regular PA, compared to the non-PA group. These results are consistent with those of previous studies demonstrating that exercise and PA help relieve mental stress in adults, adolescents, and patients with allergic diseases, such as asthma [29-31]. A prior study on 147 adolescents reported that a 10-week vigorous-intensity exercise program (30 min/session, two sessions/week) significantly reduced stress [30]. Furthermore, a study that administered a PA intervention in a school environment for low-income adolescents reported that the intervention improved the students' cardiopulmonary function $\left(\mathrm{VO}_{2 \max }\right)$ by $8.5 \%$ and reduced anxiety symptoms by $13.7 \%$ [31]. In addition, an MVPA intervention program, including psychological management, was also reported to reduce stress in undergraduate students [32]. In general, stress is associated with mental health problems, such as depression and suicide, and it may affect sleep, cardiovascular diseases, and immune system dysfunction [33]. In fact, young adults with an increased risk of mental disorder symptoms or a high incidence of mental disorders may develop serious social and occupational dysfunction [34]. Therefore, it is important to promote $\mathrm{PA}$ in adolescents with $\mathrm{AD}$, who are already at high risk of stress, to manage their mental health.

In this study, we could not examine the exact effects of regular PA on adolescents' allergic immune responses. However, we observed that regular PA was positively associated with psychological stress in the Korean adolescent population. Some previous studies have reported that PA is associated with AD symptoms, and that patients with severe AD participate in approximately $50 \%$ less PA (adjusted OR, $0.45 ; 95 \%$ CI, 0.28-0.73) [35]. In general, MVPA causes shortness of breath or sweat. In a recent study, through metabolomics analysis of sweat in patients with $\mathrm{AD}$, it was shown that the glucose concentration in sweat increases with the severity of the disease [36]. High glucose levels in sweat can delay the repair of the damaged skin barrier and promote itching. Furthermore, since patients with AD can have a warm sensation on their skin and sweat caused by PA can actually induce itching, PA may exacerbate the symptoms of AD [18]. The results of this study do not reveal whether AD patients' symptoms worsen due to sweating after PA because it was a cross-sectional study of the Korea Youth Risk Behavior Web-Based Survey. Therefore, the causal relationship between AD symptoms and sweat caused by PA in adolescents with $\mathrm{AD}$ is unknown. Thus, future studies should examine both PA promotion and symptom improvement in patients with AD.

This study has a few limitations. First, this study used nationally representative data obtained via an online survey, and thus, we could not obtain information about AD treatment history and severity. However, one strength of this study is that the study data were extracted from a sample of more than 100,000 individuals by a government agency. Second, this was a cross-sectional study; therefore, the causality between stress and PA in adolescents with AD could not be determined. However, we specified the time of diagnosis to within one year, and the survey asked about PA within the past week, which enabled an understanding of the association between the current level of stress and PA. Third, the KYRBWS used multiple-choice questions, so the exact amount of PA could not be determined. However, the survey contained separate questions for moderate PA, vigorous PA, and muscle strengthening exercises, so we were able to distinguish intensity and form of exercise.

\section{Conclusions}

In this study, adolescents with AD had a higher stress OR than adolescents without; in particular, those who had been diagnosed with $\mathrm{AD}$ within one year had a 30\% higher risk. However, adolescents with AD who regularly engaged in MVPA and muscle strengthening exercises had significantly lower stress. Thus, it would be beneficial to emphasize the importance of PA in managing mental health for adolescents with $\mathrm{AD}$ who have a high risk of stress.

Author Contributions: All authors provided substantial contributions to the conception, design, data acquisition, analysis, and interpretation of this work. S.K., J.K., and S.K.L. drafted the manuscript. All authors participated in revising the text after critical review. All authors have read and agreed to the published version of the manuscript. 
Funding: This research received no external funding.

Conflicts of Interest: The authors declare no conflict of interest.

\section{References}

1. Langan, S.M.; Irvine, A.D.; Weidinger, S. Atopic dermatitis. Lancet 2020, 396, 345-360. [CrossRef]

2. Lee, J.H.; Han, K.D.; Kim, K.M.; Park, Y.G.; Lee, J.Y.; Park, Y.M. Prevalence of Atopic Dermatitis in Korean Children Based on Data From the 2008-2011 Korean National Health and Nutrition Examination Survey. Allergy Asthma Immunol. Res. 2016, 8, 79-83. [CrossRef]

3. Boguniewicz, M.; Leung, D.Y. Recent insights into atopic dermatitis and implications for management of infectious complications. J. Allergy Clin. Immunol. 2010, 125, 4-13. [CrossRef]

4. Buys, L.M. Treatment options for atopic dermatitis. Am. Fam. Physician 2007, 75, 523-528.

5. Hansen, T.E.; Evjenth, B.; Holt, J. Increasing prevalence of asthma, allergic rhinoconjunctivitis and eczema among schoolchildren: Three surveys during the period 1985-2008. Acta Paediatr. 2013, 102, 47-52. [CrossRef]

6. Lee, J.H.; Kim, E.H.; Cho, J.; Kim, H.Y.; Suh, J.; Ahn, K.; Cheong, H.-K.; Lee, S.I. Comparison of Prevalence and Risk Factors of Atopic Dermatitis by Physical Examination and Questionnaire Survey in Elementary School Children. Pediatr. Allergy Respir. Dis. 2011, 21, 186-196. [CrossRef]

7. Hon, K.-L.E.; Tsang, Y.-C.K.; Poon, T.C.W.; Pong, N.H.; Kwan, M.; Lau, S.; Chiu, Y.-C.; Wong, H.-H.; Leung, T.F. Predicting eczema severity beyond childhood. World J. Pediatr. 2016, 12, 44-48. [CrossRef]

8. Lee, M.K.; Seo, J.-H.; Chu, H.; Kim, H.; Jang, Y.H.; Jeong, J.W.; Yum, H.Y.; Han, M.Y.; Yoon, H.J.; Cho, S.-H.; et al. Current Status of Patient Education in the Management of Atopic Dermatitis in Korea. Yonsei Med. J. 2019, 60, 694-699. [CrossRef]

9. Koszorú, K.; Borza, J.; Gulácsi, L.; Sárdy, M. Quality of life in patients with atopic dermatitis. Cutis 2019, 104, 174-177.

10. Choi, C. The factors affecting the life satisfaction of adolescents with atopic dermatitis. Stud. Korean Youth 2015, 26, 111-144.

11. Schut, C.; Weik, U.; Tews, N.; Gieler, U.; Deinzer, R.; Kupfer, J. Psychophysiological effects of stress management in patients with atopic dermatitis: A randomized controlled trial. Acta Derm. Venereol. 2013, 93, 57-61. [CrossRef] [PubMed]

12. Buske-Kirschbaum, A.; Geiben, A.; Hellhammer, D. Psychobiological aspects of atopic dermatitis: An overview. Psychother. Psychosom. 2001, 70, 6-16. [CrossRef] [PubMed]

13. Sandhu, J.K.; Wu, K.K.; Bui, T.L.; Armstrong, A.W. Association between Atopic Dermatitis and Suicidality: A Systematic Review and Meta-analysis. JAMA Dermatol. 2019, 155, 178-187. [CrossRef] [PubMed]

14. Kwon, J.A.; Park, E.C.; Lee, M.; Yoo, K.B.; Park, S. Does stress increase the risk of atopic dermatitis in adolescents? Results of the Korea Youth Risk Behavior Web-based Survey (KYRBWS-VI). PLoS ONE 2013, 8, e67890. [CrossRef]

15. Janssen, I. Physical activity guidelines for children and youth. Can. J. Public Health 2007, 98 (Suppl. 2), S109-S121.

16. Pascoe, M.C.; Bailey, A.P.; Craike, M.; Carter, T.; Patten, R.; Stepto, N.; Parker, A. Physical activity and exercise in youth mental health promotion: A scoping review. BMJ Open Sport Exerc. Med. 2020, 6, e000677. [CrossRef]

17. Son, W.K.; Yoon, W.; Kim, S.; Byeon, J.H.; Lee, J.S.; Kim, D.; Jaehoon, L.; Chae, Y.; Yoon, S.J.; Yoo, Y. Can moderate-intensity aerobic exercise ameliorate atopic dermatitis? Exp. Dermatol. 2020. [CrossRef]

18. Murota, H.; Yamaga, K.; Ono, E.; Murayama, N.; Yokozeki, H.; Katayama, I. Why does sweat lead to the development of itch in atopic dermatitis? Exp. Dermatol. 2019, 28, 1416-1421. [CrossRef]

19. Lim, M.S.; Lee, C.H.; Sim, S.; Hong, S.K.; Choi, H.G. Physical Activity, Sedentary Habits, Sleep, and Obesity are Associated with Asthma, Allergic Rhinitis, and Atopic Dermatitis in Korean Adolescents. Yonsei Med. J. 2017, 58, 1040-1046. [CrossRef]

20. Silverberg, J.I.; Song, J.; Pinto, D.; Yu, S.H.; Gilbert, A.L.; Dunlop, D.D.; Chang, R.W. Atopic Dermatitis is Associated with Less Physical Activity in US Adults. J. Investig. Dermatol. 2016, 136, 1714-1716. [CrossRef]

21. Twisk, J.W.R. Physical Activity Guidelines for Children and Adolescents. Sports Med. 2001, 31, 617-627. [CrossRef] 
22. Park, H.; Kim, K. Association of Perceived Stress with Atopic Dermatitis in Adults: A Population-Based Study in Korea. Int. J. Environ. Res. Public Health 2016, 13, 760. [CrossRef]

23. Rod, N.H.; Kristensen, T.S.; Lange, P.; Prescott, E.; Diderichsen, F. Perceived stress and risk of adult-onset asthma and other atopic disorders: A longitudinal cohort study. Allergy 2012, 67, 1408-1414. [CrossRef] [PubMed]

24. Arndt, J.; Smith, N.; Tausk, F. Stress and atopic dermatitis. Curr. Allergy Asthma Rep. 2008, 8, 312-317. [CrossRef]

25. Bauman, A.E. Updating the evidence that physical activity is good for health: An epidemiological review 2000-2003. J. Sci. Med. Sport 2004, 7, 6-19. [CrossRef]

26. Biddle, S.J.H.; Asare, M. Physical activity and mental health in children and adolescents: A review of reviews. Br. J. Sports Med. 2011, 45, 886-895. [CrossRef]

27. Wheatley, C.; Wassenaar, T.; Salvan, P.; Beale, N.; Nichols, T.; Dawes, H.; Johansen-Berg, H. Associations between fitness, physical activity and mental health in a community sample of young British adolescents: Baseline data from the Fit to Study trial. BMJ Open Sport Exerc. Med. 2020, 6, e000819. [CrossRef]

28. Mücke, M.; Ludyga, S.; Colledge, F.; Gerber, M. Influence of Regular Physical Activity and Fitness on Stress Reactivity as Measured with the Trier Social Stress Test Protocol: A Systematic Review. Sports Med. 2018, 48, 2607-2622. [CrossRef] [PubMed]

29. O’Dougherty, M.; Hearst, M.O.; Syed, M.; Kurzer, M.S.; Schmitz, K.H. Life events, perceived stress and depressive symptoms in a physical activity intervention with young adult women. Ment. Health Phys. Act. 2012, 5, 148-154. [CrossRef]

30. Norris, R.; Carroll, D.; Cochrane, R. The effects of physical activity and exercise training on psychological stress and well-being in an adolescent population. J. Psychosom. Res. 1992, 36, 55-65. [CrossRef]

31. Bonhauser, M.; Fernandez, G.; Püschel, K.; Yañez, F.; Montero, J.; Thompson, B.; Coronado, G. Improving physical fitness and emotional well-being in adolescents of low socioeconomic status in Chile: Results of a school-based controlled trial. Health Promot. Int. 2005, 20, 113-122. [CrossRef]

32. Baghurst, T.; Kelley, B.C. An examination of stress in college students over the course of a semester. Health Promot. Pract. 2014, 15, 438-447. [CrossRef] [PubMed]

33. Stults-Kolehmainen, M.A.; Sinha, R. The effects of stress on physical activity and exercise. Sports Med. 2014, 44, 81-121. [CrossRef]

34. Scott, E.M.; Hermens, D.F.; Glozier, N.; Naismith, S.L.; Guastella, A.J.; Hickie, I.B. Targeted primary care-based mental health services for young Australians. Med. J. Aust. 2012, 196, 136-140. [CrossRef]

35. Strom, M.A.; Silverberg, J.I. Associations of Physical Activity and Sedentary Behavior with Atopic Disease in United States Children. J. Pediatrics 2016, 174, 247-253.e243. [CrossRef]

36. Ono, E.; Murota, H.; Mori, Y.; Yoshioka, Y.; Nomura, Y.; Munetsugu, T.; Yokozeki, H.; Katayama, I. Sweat glucose and GLUT2 expression in atopic dermatitis: Implication for clinical manifestation and treatment. PLOS ONE 2018, 13, e0195960. [CrossRef]

Publisher's Note: MDPI stays neutral with regard to jurisdictional claims in published maps and institutional affiliations.

(C) 2020 by the authors. Licensee MDPI, Basel, Switzerland. This article is an open access article distributed under the terms and conditions of the Creative Commons Attribution (CC BY) license (http://creativecommons.org/licenses/by/4.0/). 